\title{
Phenotyping GABA transaminase deficiency: a case description and literature review
}

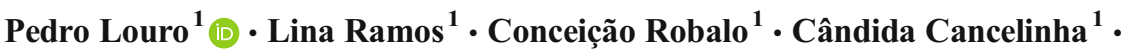 \\ Alexandra Dinis ${ }^{1} \cdot$ Ricardo Veiga $^{1}$ - Raquel Pina ${ }^{1}$ - Olinda Rebelo ${ }^{1}$ - Ana Pop ${ }^{2}$. \\ Luísa Diogo $^{1} \cdot$ Gajja S. Salomons $^{2} \cdot$ Paula Garcia $^{1}$
}

Received: 19 April 2016/Revised: 20 May 2016/Accepted: 30 May 2016/Published online: 4 July 2016

(C) SSIEM 2016

\begin{abstract}
Gamma-aminobutyric acid transaminase (GABA$\mathrm{T})$ deficiency is an autosomal recessive disorder reported in only three unrelated families. It is caused by mutations in the $A B A T$ gene, which encodes 4-aminobutyrate transaminase, an enzyme of GABA catabolism and mitochondrial nucleoside salvage. We report the case of a boy, deceased at 12 months of age, with early-onset epileptic encephalopathy, severe psychomotor retardation, hypotonia, lower-limb hyporeflexia, central hypoventilation, and rapid increase in weight and, to a lesser rate, length and head circumference. He presented signs of premature pubarche, thermal instability, and water-electrolyte imbalance. Serum total testosterone was elevated (43.3 ng/dl; normal range $<16$ ), as well as serum growth hormone $(7.7 \mathrm{ng} / \mathrm{ml}$; normal range $<1)$. Brain magnetic resonance imaging (MRI) showed decreased myelination and generalized brain atrophy, later confirmed by post-mortem examination. $A B A T$ gene sequencing was performed post-mortem, identifying a homozygous variant c. $888 \mathrm{G}>\mathrm{T}$ (p.Gln296His),not previously described. In vitro analysis concluded that this variant is pathogenic. The clinical features of this patient are similar to those reported so far in GABA-T deficiency. However, distinct mutations may have a different effect on enzymatic
\end{abstract}

Communicated by: Jaak Jaeken

Pedro Louro

pjplouro@gmail.com

1 Centro Hospitalar e Universitário de Coimbra, Praceta Prof. Mota Pinto, 3000-075 Coimbra, Portugal

2 Metabolic Unit, Department of Clinical Chemistry, VU University Medical Center, Neuroscience Campus Amsterdam,

Amsterdam, The Netherlands activity, which potentially could lead to a variable clinical outcome. Clinical investigation aiming for a diagnosis should not end with the patient's death, as it may allow a more precise genetic counselling for the family.
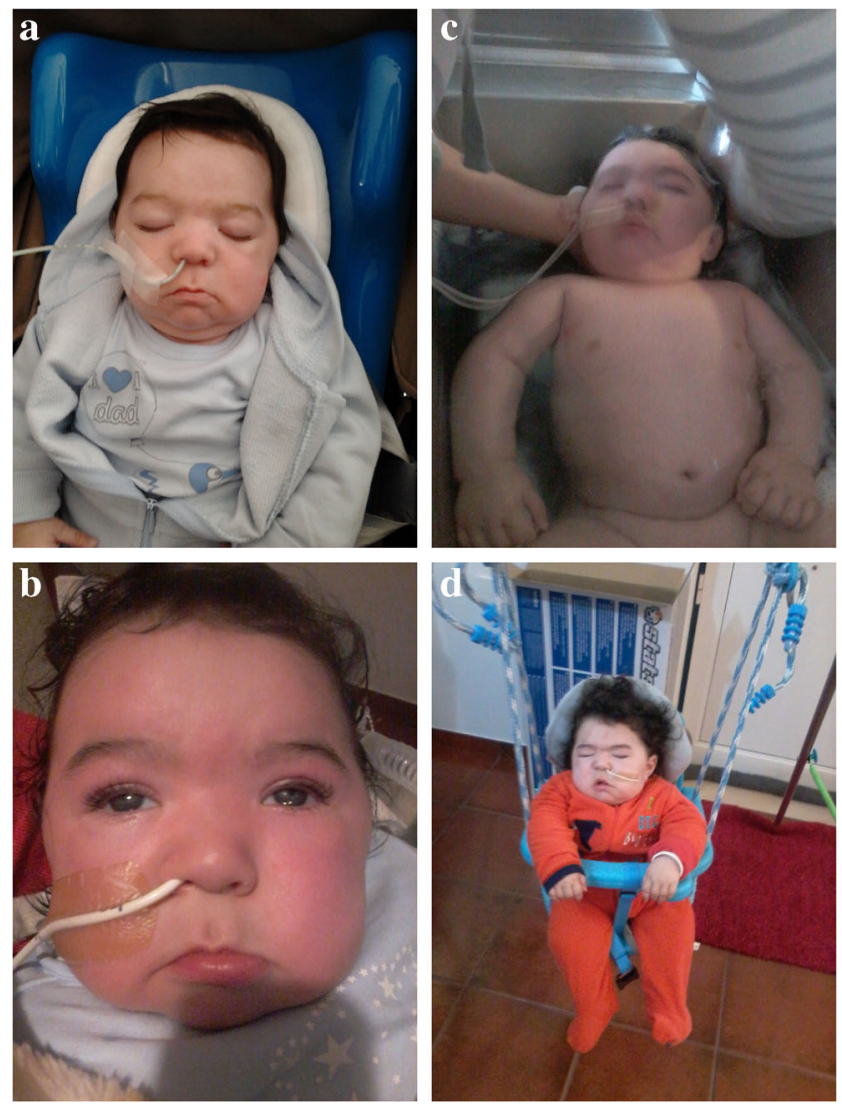

Fig. 1 Patient's photos (note the increasingly "puffy" appearance) at 2 months (a), 5 months (b), 10 months (c), and 11 months (d) of age 
Fig. 2 Patient's weight (a),

length (b), and head

circumference growth (c) (growth

charts reproduced with

permission from World Health

Organization)
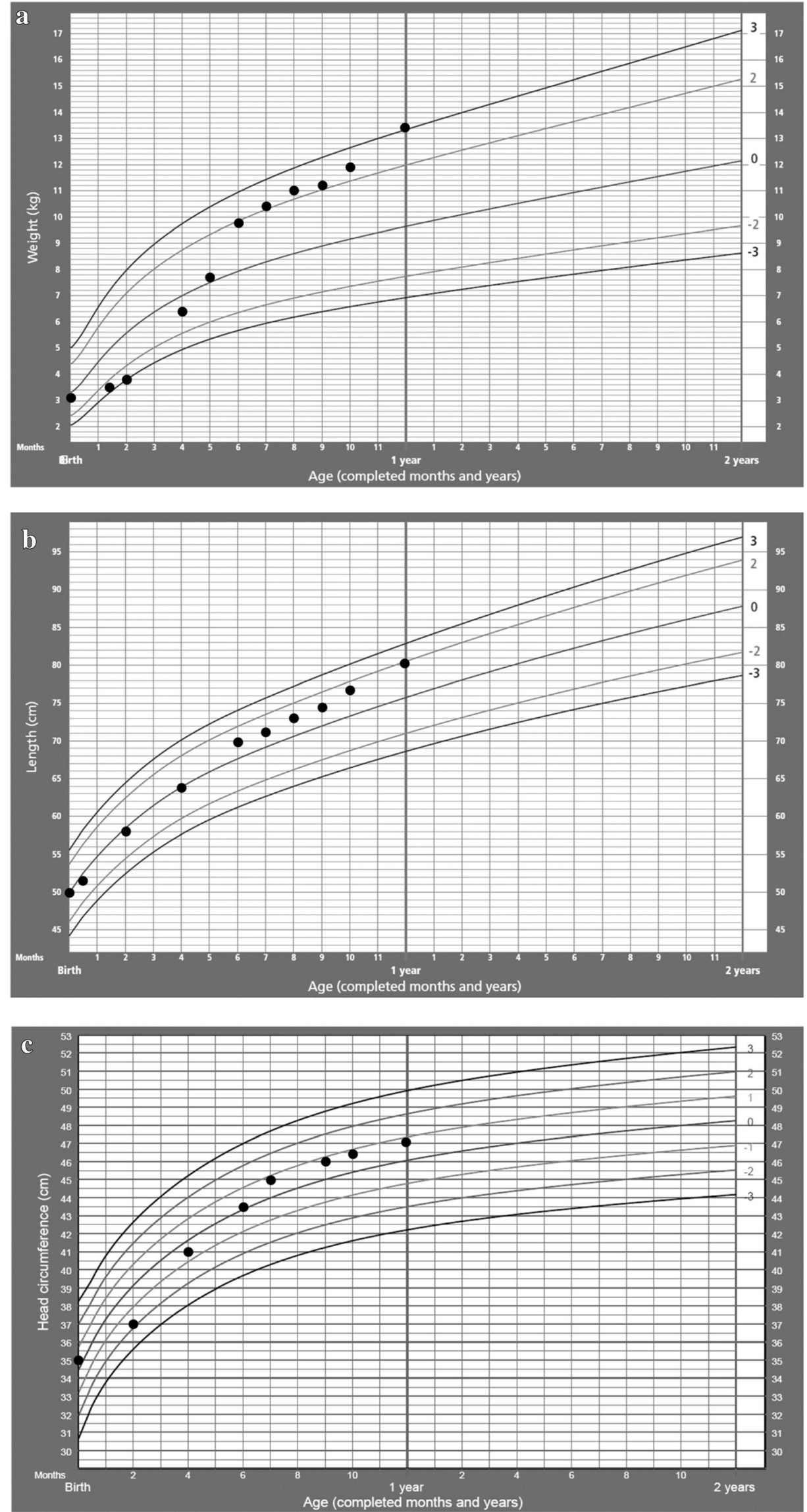


\section{Introduction}

Gamma-aminobutyric acid transaminase (GABA-T) deficiency (MIM\#613163) is an autosomal recessive disorder reported in only three unrelated families (Jaeken et al 1984; Tsuji et al 2010; Besse et al 2015). It is caused by mutations in the $A B A T$ gene (HGNC_ID:23), which encodes 4-aminobutyrate transaminase (EC 2.6.1.19), an enzyme of GABA catabolism and mitochondrial nucleoside salvage (Besse et al 2015). GABA$\mathrm{T}$ deficiency is usually characterized by elevated levels of GABA in cerebrospinal fluid (CSF), associated with severe psychomotor retardation, intractable seizures, hypotonia with hyperreflexia, accelerated height growth, and a high-pitched cry (Jaeken et al 1984; Tsuji et al 2010; Besse et al 2015).

\section{Case report}

The patient (Fig. 1) was a Portuguese male infant, born full term by caesarean delivery due to cephalopelvic disproportion. He was the first child of healthy parents. Consanguinity was denied but both parents originate from the same Portuguese region. He was admitted at the age of 3 days for generalized hypotonia since birth, progressing to encephalopathy and coma. Electroencephalography (EEG) showed a burst-suppression pattern compatible with early-onset epileptic encephalopathy. Levetiracetam was started, initially reducing the frequency of seizures, but ultimately with no effect. Until his death at the age of 12 months, he presented severe psychomotor retardation, lower-limb hyporeflexia, central hypoventilation, bilateral cryptorchidism, and a rapid increase in weight, as well as in length and head circumference, although to a lesser rate (Fig. 2). He had a general "puffy" appearance, signs of premature pubarche, thermal instability, and water-electrolyte imbalance. He needed noninvasive ventilation and tube feeding.

Serum total testosterone was elevated $(43.3 \mathrm{ng} / \mathrm{dl}$; normal range $<16)$, as was serum growth hormone $(7.7 \mathrm{ng} / \mathrm{ml}$; normal range $<1$ ). Brain magnetic resonance imaging (MRI) showed decreased myelination and generalized cerebral, cerebellar, and brainstem atrophy, later confirmed by post-mortem examination. Diffusion-weighted images revealed bilateral hyperintense areas involving subcortical and periventricular white matter, and globus pallidus (Fig. 3). Magnetic resonance spectroscopy (MRS) showed no abnormalities. Neuropathological examination revealed soft consistency of the white matter. Microscopically, subcortical white matter is seen as a palestained/poorly myelinated area on the slide (Fig. 4).

The diagnosis of GABA-T deficiency was considered postmortem. $A B A T$ gene sequencing identified a homozygous variant c.888G $>$ T (p.Gln296His), previously undescribed. MutationTaster, PolyPhen2 and SIFT predict this variant to be, respectively, "disease causing", "probably damaging"

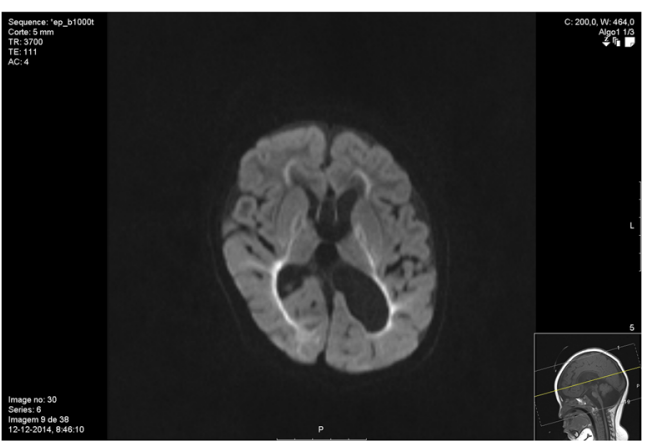

Fig. 3 Brain magnetic resonance imaging (MRI) at 12 months of age. Diffusion-weighted axial images show bilateral hyperintense areas involving subcortical and periventricular white matter, and globus pallidus

and "damaging". In vitro analysis concluded that this variant is pathogenic (Pop et al 2015). At the time of the diagnosis, the patient's parents had an 11-weeks' gestation. Chorionic villus sampling was performed, and molecular analysis confirmed that the fetus was not homozygous for the pathogenic variant.

\section{Discussion}

The clinical features of this patient are similar to those reported in GABA-T deficiency cases (Table 1). Interestingly,

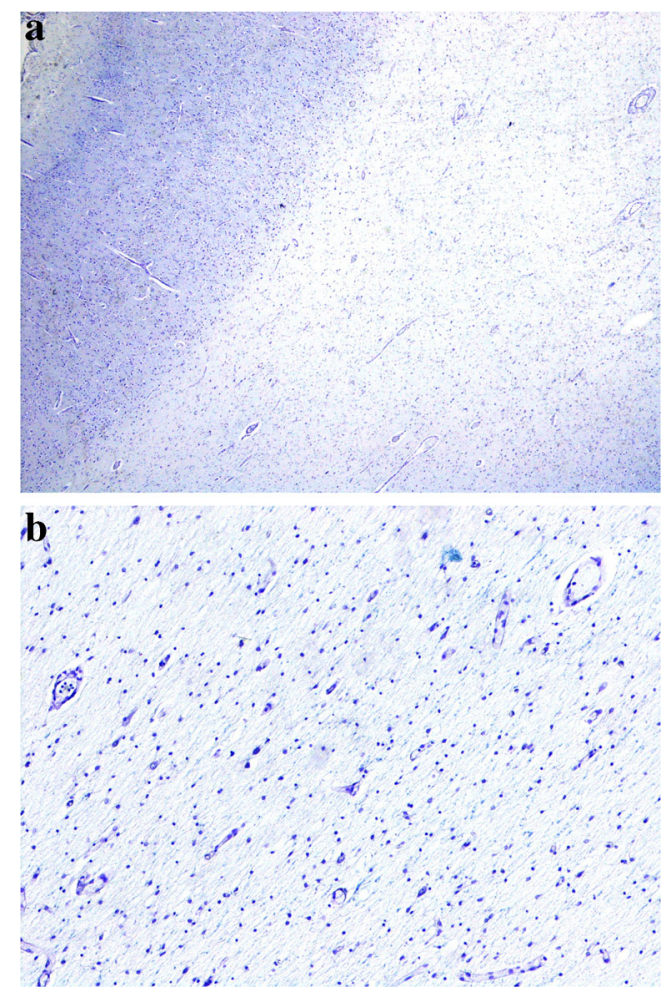

Fig. 4 Cerebral cortex and poorly myelinated subcortical white matter (Paraffin section, Klüver-Barrera staining, ×40) (a). Poorly myelinated subcortical white matter (Paraffin section, Klüver-Barrera staining, $\times 200)(\mathbf{b})$ 


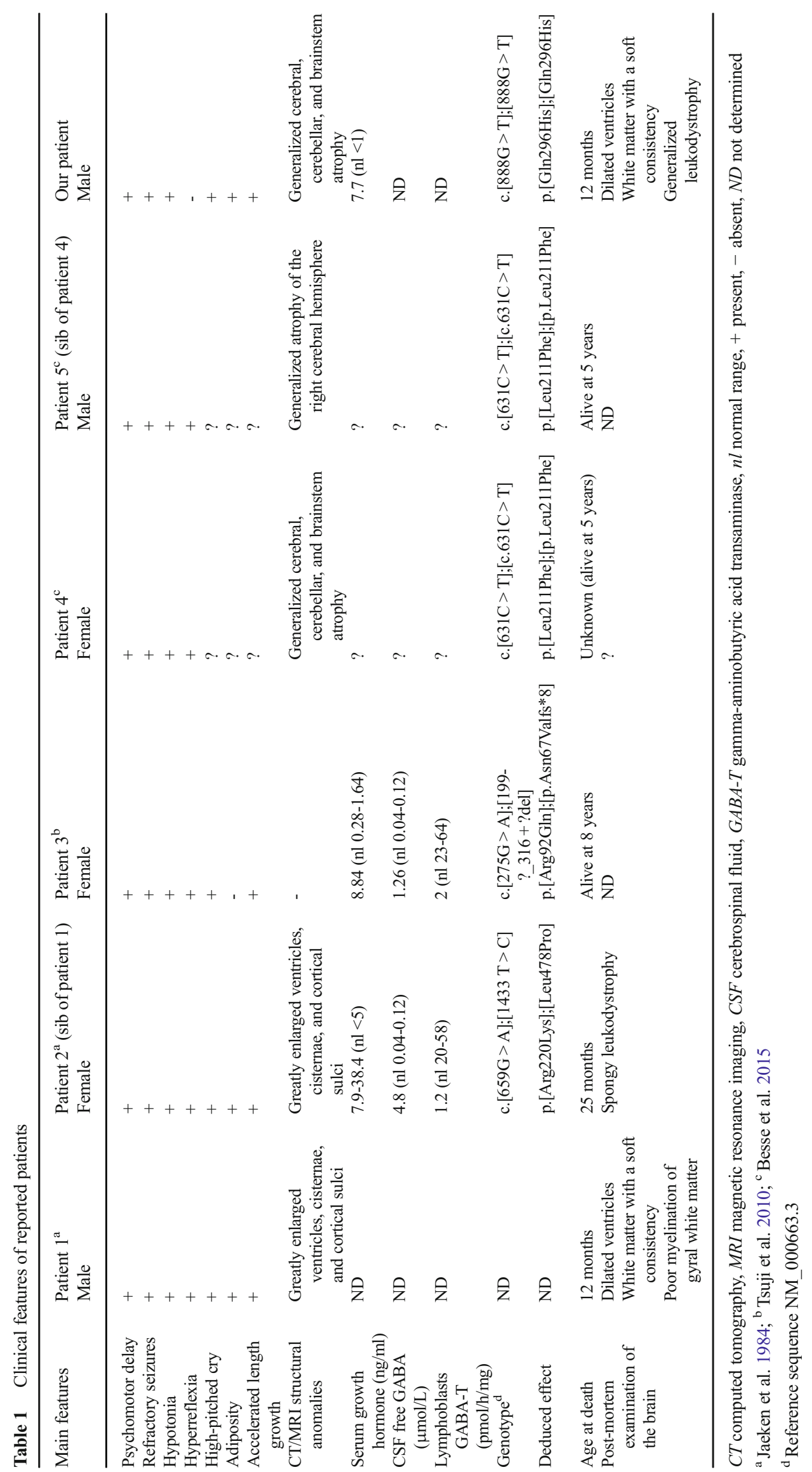


hyperreflexia was not present in our patient. His weight gain almost overshadowed length growth. These growth alterations seem to be related with the abnormally high levels of growth hormone, while the signs of premature pubarche are likely to be related with the high testosterone levels, which may also have contributed to the patient's "puffy" appearance. High GABA levels probably contributed to this hormone imbalance (Jaeken et al 1990; Ritta et al 1998).

Certain mutations express a residual enzymatic activity (Pop et al 2015), which potentially could lead to a variable clinical outcome. However, other (epi)genetic factors could also have an effect. CSF amino acid analysis was performed but did not include free GABA measurement; when GABA-T deficiency was considered, it could not be performed due to the lack of a CSF sample. This reinforces the need for the rigorous implementation of sample collection and storing protocols, mostly in severely affected patients, during life and even at post-mortem. However, lumbar puncture is an invasive procedure, and sampling is not always easy. In our case, collected CSF volume was not enough to store.

Detection of brain GABA elevation by MRS is difficult and usually requires a technical modification (Levy and Degnan 2013), which was not considered in this patient while he was alive. GABA-T is also involved in the mitochondrial nucleoside salvage pathway. Mitochondrial DNA (mtDNA) copy number measurement was not performed in our case, but an mtDNA depletion was previously demonstrated in fibroblasts from other patients (Besse et al. 2015). Evidence of a depletion would probably have misled us to disorders with phenotypic overlap, such as the mtDNA depletion syndromes caused by mutations in SUCLG1 and SUCLA2 genes (Besse et al 2015). Clinical investigation aiming a diagnosis should not end with the patient's death, as it may allow a more precise genetic counselling for the families. In our case, it allowed us to offer our patient's parents a molecular prenatal diagnosis.

Acknowledgments We thank Dr. Megumi Tsuji (Kanagawa Children's Medical Centre, Japan) for her kind help.

\section{Compliance with ethical standards}

Conflict of interest None.

\section{References}

Besse A, Wu P, Bruni F, Donti T, Graham BH, Craigen WJ et al (2015) The GABA transaminase, ABAT, is essential for mitochondrial nucleoside metabolism. Cell Metab 21(3):417-427

Jaeken J, Casaer P, de Cock P, Corbeel L, Eeckels R, Eggermont E et al (1984) Gamma-aminobutyric acid-transaminase deficiency: a newly recognized inborn error of neurotransmitter metabolism. Neuropediatrics 15(3):165-169

Jaeken J, Casaer P, Haegele KD, Schechter PJ (1990) Review: normal and abnormal central nervous system GABA metabolism in childhood. J Inherit Metab Dis 13(6):793-801

Levy LM, Degnan AJ (2013) GABA-based evaluation of neurologic conditions: MR spectroscopy. Am J Neuroradiol 34(2):259-265

Pop A, Janssen EAW, Roos B, Struys EA, Oostendorp J, Louro P et al (2015) Model system for fast in vitro analysis of GABA-T missense variants [abstract]. J Inherit Metab Dis 38(Suppl 1):S315-S316

Ritta MN, Calamera JC, Bas DE (1998) Occurrence of GABA and GABA receptors in human spermatozoa. Mol Hum Reprod 4(8): 769-773

Tsuji M, Aida N, Obata T, Tomiyasu M, Furuya N, Kurosawa K et al (2010) A new case of GABA transaminase deficiency facilitated by proton MR spectroscopy. J Inherit Metab Dis 33(1):85-90 